\title{
A MICRO GAS CHROMATOGRAPHY COLUMN WITH AN EMBEDDED OUT-OF-PLANE THERMAL CONDUCTIVITY DETECTOR
}

\author{
Shree Narayanan and Masoud Agah
}

VT MEMS Laboratory, Bradley Department of Electrical and Computer Engineering, Virginia Tech, USA

\begin{abstract}
We present the design, fabrication and experimental evaluation of a unique micro gas chromatography ( $\mu \mathrm{GC})$ column with an integrated micro thermal conductivity detector ( $\mu \mathrm{TCD})$. The resistors are dry released to form $170 \mu \mathrm{m}$ diameter coils. The high isolation of the sensing element provides for an improved sensitivity by an order of magnitude over our previously reported designs. The chip incorporates a 2-port design approach and a device size of $2 \mathrm{~cm} \times 4 \mathrm{~cm}$. Fabricated using a four-mask process, the resistors and microfluidics are independently processed on borofloat and silicon wafers, respectively, and subsequently anodic bonded. The separation column is static coated without damage to the resistors. The coated column-detector chip has successfully separated a representative hydrocarbon mixture in less than 2 minutes while consuming only $50 \mathrm{~mW}$ of power for TCD operation.
\end{abstract}

\section{INTRODUCTION}

Gas chromatography (GC), the technique of separating a mixture of gases into its components, has applications ranging across space exploration, homeland security, dissolved gas analysis and food quality testing [1]. Conventional gas chromatography is an established field that is more suited to lab testing owing to large bulky instruments that consume large power. Micro gas chromatography $(\mu \mathrm{GC})$ refers to the miniaturization of a $\mathrm{GC}$ system using microfabrication techniques. The resulting advantages include lower power consumption and batch fabrication of components that reduces cost. In addition, their light weight enables field-use. Microfabrication allows the development of innovative structures and geometries to test the theoretical extremes of optimized performance that might otherwise be difficult in conventional GC $[2,3]$.

Multiple efforts have been invested into the development of the individual components of a $\mu \mathrm{GC}$ system and their integration. Some noteworthy attempts include the hybrid integration of detectors reported in $[4,5]$. However, the effort towards monolithic integration of detectors with separation column has been minimal and needs further investigation [6, 7]. Previously, we have reported the integration of a TCD with a separation column, in a unique configuration that saved space, reduced the number of fluidic interconnections, and at the same time provided for the thermostatted operation of the separation column [8].

In this paper, we present an improved version of the thermal conductivity detector wherein the resistors are high aspect ratio (HAR) fabricated with a modified silicon sacrificial layer dry etch (SSLDE) process. These released structures were anodic bonded with the separation column and resulted in an order of magnitude increased sensitivity while requiring only $50 \mathrm{~mW}$ for operation.

\section{THEORY \\ GC System}

A GC system comprises of three main components viz., preconcentrator, separation column, and detector. The preconcentrator is responsible for amplifying the compounds of interest to a level that will exceed the limit of detection of the detector $[9,10]$. The concentrated sample from the preconcentrator is injected into a column whose task is to separate the gases. In its conventional form, a separation column is a capillary whose internal surface is coated with a compound referred to as the stationary phase. As the mixture is carried along by a carrier gas through the capillary, different compounds interact with the stationary phase to a different extent resulting in separation. In the microfabrication world, these capillaries are typically channels etched in silicon. The eluted compounds then pass into a detector such as a flame ionization detector (FID), chemiresistors, a thermal conductivity detector (TCD), or a surface acoustic wave (SAW) sensor.

\section{Monolithic Integration of Detector}

We have opted to integrate a TCD, owing to its universal detection capability, simplicity and robustness. It consists of a heated resistor placed in a carrier gas which is often helium due to its high thermal conductivity $(0.1513 \mathrm{~W} / \mathrm{m} . \mathrm{K})$. When a component of differing thermal conductivity comes in contact with the resistor, the heat flux gradient is altered and hence, the temperature of the resistor changes. By measuring this change as a differential measurement against a reference resistor, which does not come in contact with the eluted compound, a signal corresponding to the eluted compound is obtained. We have previously shown a technique to place the reference resistor close to the inlet of the separation column and detect the compound, though with limited sensitivity [11]. By suspending the resistors into the gas stream, the sensitivity of detection can be increased [12].

Prior reports in literature to suspend the resistor have employed under-etching thin metal films on silicon to simultaneously form the fluidic channel [7, 13]. This limits our ability to manipulate the silicon substrate. Further, the Borofloat lid remains unused. In this paper, we have released a resistor which is deposited on Borofloat, and yet released, leaving the silicon free to be etched to our needs. In particular we have used a modified form of the sacrificial silicon layer dry etch (SSLDE) technique to ensure that in addition to the release, the Borofloat is still suitable to be bonded to the silicon separation column [14].

\section{FABRICATION}

The device consists of a silicon substrate hosting the separation microfluidic channel and a Borofloat wafer hosting the TCD which are separately processed and finally bonded together. The process flow is summarized in Fig. 1.

A $700 \mu \mathrm{m}$ thick Borofloat wafer is blanket deposited with a 650nm thick sacrificial PECVD oxide. AZ5214 was spun coated and patterned in image reversal mode to define the anchor. A 200nm thick sacrificial silicon was e-beam evaporated and lifted off on the patterned AZ5214. Another 1.5 $\mu \mathrm{m}$ thick "structural" PECVD oxide was then blanket deposited. Following this, a $60 \mathrm{~nm} / 70 \mathrm{~nm}$ thick $\mathrm{Cr} / \mathrm{Ni}$ metal stack was evaporated and lifted off using the mask of the resistor. A $1.2 \mu \mathrm{m}$ thick AZ5214, patterned in image reversal mode, on the metallic resistor served as the mask for further processing. The wafer was diced prior to the releasing step. The releasing step consisted of an initial 5 minute wet etch of the structural oxide in Buffered Oxide Etch (BOE), revealing the sacrificial silicon. The device was then mounted on a handle wafer and the sacrificial silicon etched away using the SSLDE process 
[14]. This was performed in an Alcatel AMS100 i-Speeder DRIE, with $0.1 \mathrm{mbar}$ chamber pressure, $1500 \mathrm{~W}$ coil power, no substrate bias at $20^{\circ} \mathrm{C}$, platen height of $200 \mathrm{~mm}$, and a $\mathrm{SF}_{6}$ flow rate of $300 \mathrm{sccm}$. The chemistry of $\mathrm{SF}_{6}$ enables high lateral etch rates of up to $15 \mu \mathrm{m} / \mathrm{min}$. In the absence of the sacrificial PECVD oxide, the Borofloat surface is exposed to the aggressive $\mathrm{SF}_{6}$ plasma, while the resistors are being under etched. The surface is then rendered unsuitable for anodic bonding. Upon release, the device is raised to $400^{\circ} \mathrm{C}$ on a hot plate in atmosphere. This step anneals the thin metal film and causes further curling, giving the active element of the resistors a coil shape as shown in Fig. 2. The coil has a cross sectional diameter of $168 \mu \mathrm{m}$, giving it sufficient coverage over the entire fluidic channel cross-section. which is $400 \mu \mathrm{m}$ wide and $250 \mu \mathrm{m}$ deep. The interconnects and bond pads are anchored to the substrate. The photoresist was then ashed in an oxygen plasma.
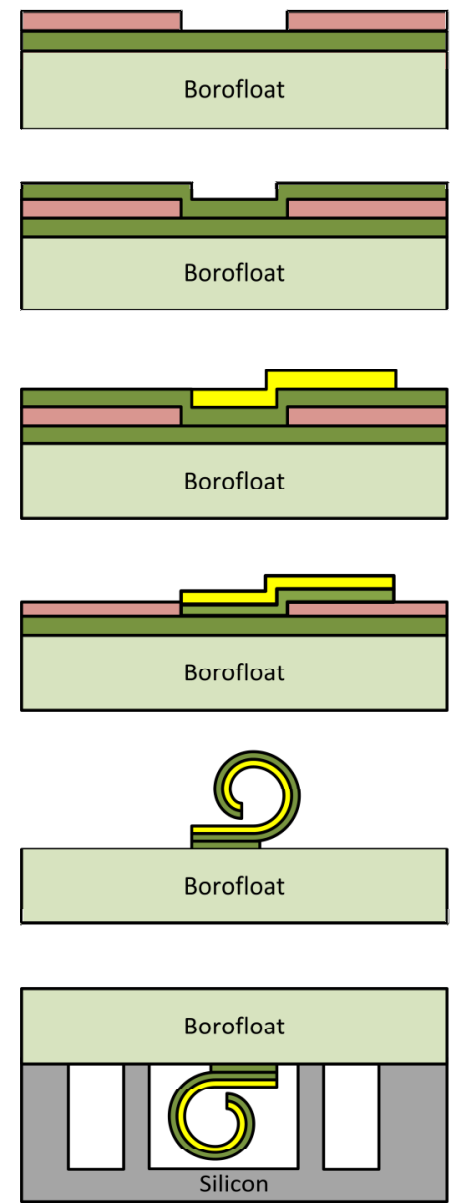

Figure 1: Fabrication of released coiled metal resistors by sacrificial etch of silicon. The photoresist used as a mask to protect the metal while under-etching has been avoided in the diagram, for simplicity

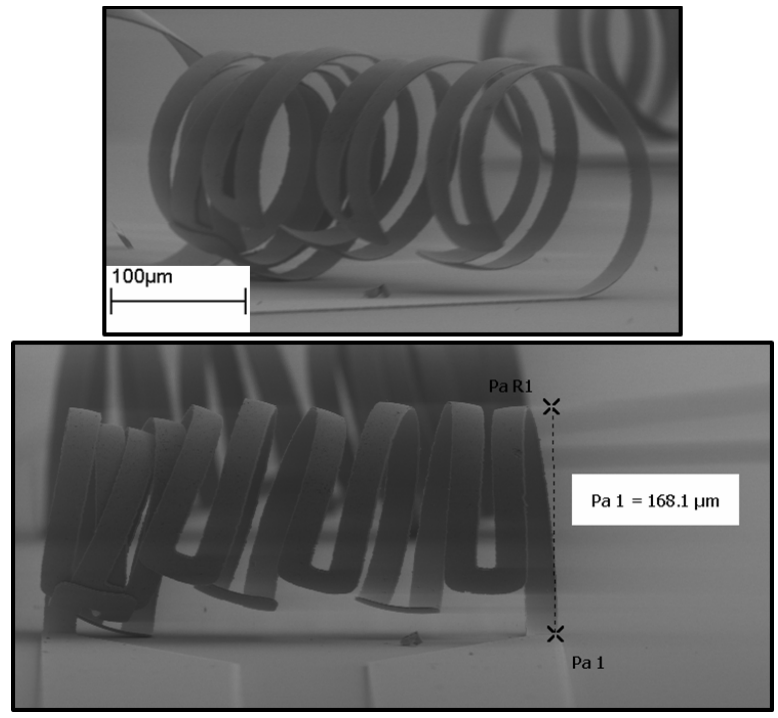

Figure 2: SEM image showing a side view (on top) and the front view (bottom) of the released coils.

The separation column was fabricated on a $500 \mu \mathrm{m}$ thick single-side polished silicon wafer. First, the wafer was spun coated with S1813. The photoresist was then patterned and etched to a shallow depth of $2-3 \mu \mathrm{m}$. This shallow etch prevents contact between the metal interconnects on the Borofloat wafer and the walls of the separation column in silicon, upon bonding. The photoresist was stripped and the wafer spun coated with AZ9260. The $8 \mu \mathrm{m}$-thick photoresist was patterned with a mask for subsequent deep etching of the channels. The Alcatel DRIE was employed in an anisotropic process, resulting in $250 \mu \mathrm{m}$-deep channels for the separation column. Finally, the photoresist was stripped off, and the silicon wafer also diced into individual devices.

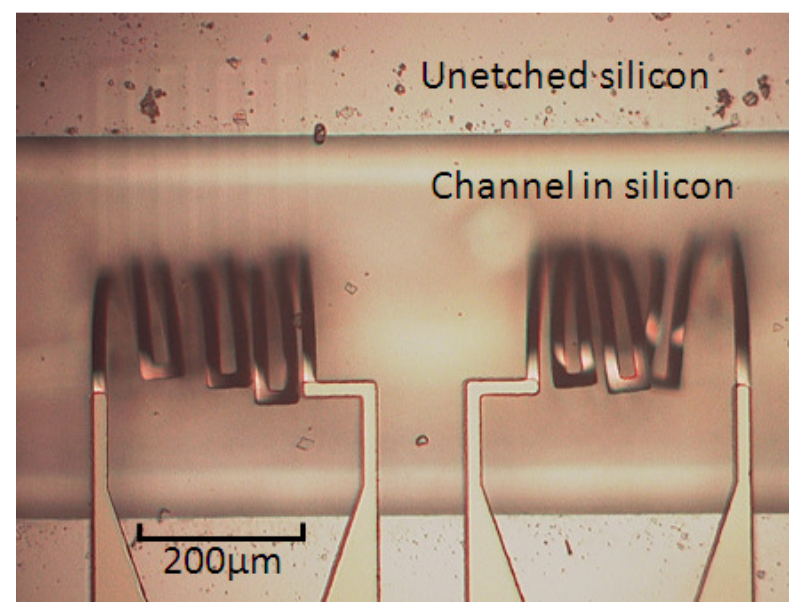

Figure 3: View of the released structures within the channel in a bonded device.

The diced detector on Borofloat and the diced separation column on silicon were aligned and brought into contact using a pick and place bonder followed by anodic bonding. Fig 3 shows the alignment of the resistors in the fluidic channel, while seeing through the Borofloat wafer. The coiled structures are mostly outof-focus due the out-of-plane bending. They align into the fluidic channel etched in silicon, which acts as a recess. 
Capillary tubes were fixed to the inlet and outlet ports of the device. The device was then static coated with polydimethylsiloxane [15]. This was achieved by filling the channel with a solution of polydimethylsiloxane (OV1) in pentane at 40psi followed by drawing vacuum at one end with the other end sealed with wax. Vaporization of pentane leaves behind a thin film of polydimethylsiloxane which serves as the stationary phase. It should be noted that the flow of the viscous solution through the resistors did not cause the released structures to detach, at the point of anchor. This is a current point of investigation to understand the limit of fluidic flow, which will cause the resistors to detach. An optical image of the packaged device is shown in Fig 4.

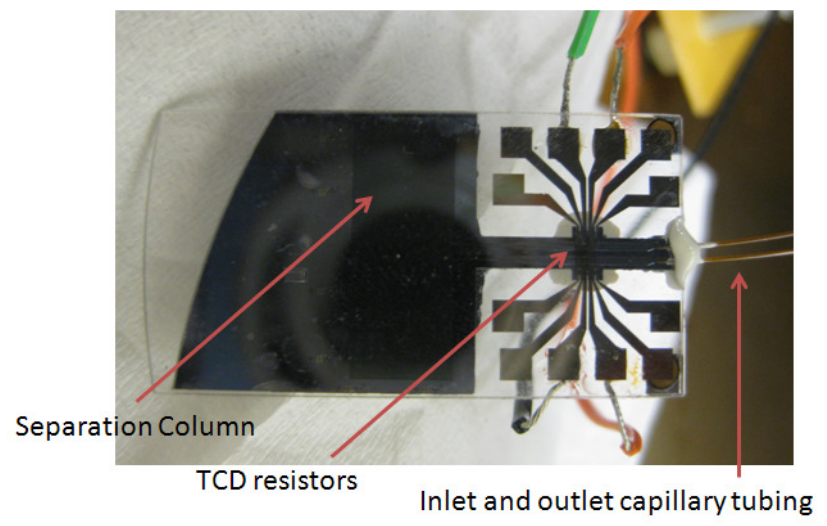

Figure 4: Optical image of the packaged device.

\section{RESULTS AND DISCUSSSION}

A $1 \mathrm{~m}$-long, 100 $\mu \mathrm{m}$-wide separation column was fabricated with the released resistors and packaged as show in Fig 5. A current of $10 \mathrm{~mA}$ was sourced into a Wheatstone bridge with the two resistors in each of its arms. The differential voltage was fed into an Agilent 34401A and recorded through LabVIEW. The device was installed in an HP5890. The injector inlet of the HP5890 was maintained at 11 psi of Helium@ $280^{\circ} \mathrm{C}$ while the oven itself was held at $65^{\circ} \mathrm{C}$. The device was tested by injecting a $0.2 \mu \mathrm{l}$ sample from a mixture of $20 \mu \mathrm{l}$ each of n-nonane, $\mathrm{n}$-decane, $\mathrm{n}$-undecane and $\mathrm{n}$-dodecane diluted in $200 \mu \mathrm{l}$ of n-octane. The response from the TCD is shown in Fig. 5. The suspended nature of the resistors resulted in a reduction in power by a factor of four. Additionally, our non-suspended TCD could barely detect $5000 \mathrm{ppm}$ of pentane in octane while we could readily achieve $500 \mathrm{ppm}$ sensitivity with our new released resistors.

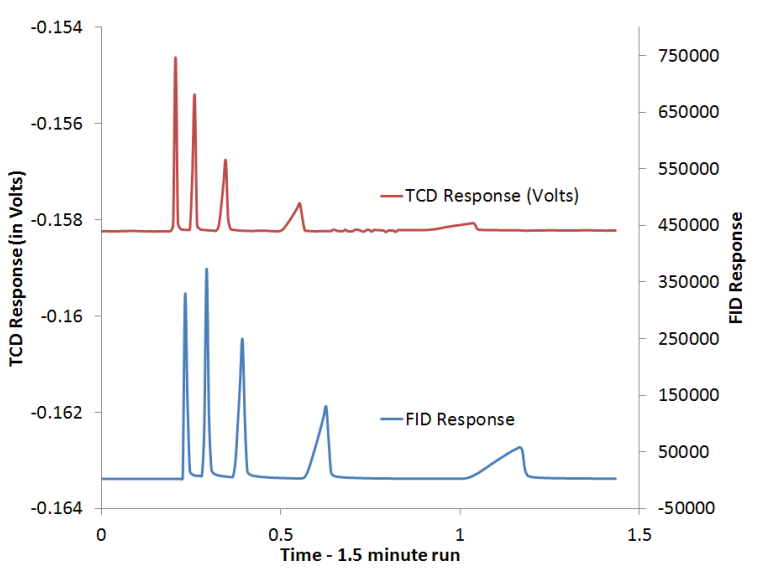

Figure 5: Comparison of the TCD and FID response to a mixture of n-hexane, $n$-octane, $n$-nonane, $n$-decane and $n$ undecane.

\section{ACKNOWLEDGEMENTS}

This work has been supported primarily by the National Science Foundation CAREER award (ECCS-0747600). Travel support has ben generously provided by the Transducer Research Foundation. The authors would like to thank Mr. Don Leber with the Virginia Tech MicrOn cleanroom for his technical assistance and the staff at Nanoscale Characterization and Fabrication Laboratory (NCFL).

\section{REFERENCES}

[1] G. Monti, et al., "Monitoring Food Quality by Microfluidic Electrophoresis, Gas Chromatography, and Mass Spectrometry Techniques: Effects of Aquaculture on the Sea Bass (Dicentrarchus labrax)," Analytical Chemistry, vol. 77, pp. 2587-2594, 2005/04/01 2005.

[2] H. Shakeel, et al., "First reconfigurable MEMS separation columns for micro gas chromatography," 2012, pp. 823-826.

[3] B. Alfeeli, et al., "Low pressure drop micro preconcentrators with cobweb Tenax-TA film for analysis of human breath," 2011, pp. 916-919.

[4] S. K. Kim, et al., "Microfabricated Gas Chromatograph for the Selective Determination of Trichloroethylene Vapor at Sub-Parts-Per-Billion Concentrations in Complex Mixtures," Analytical Chemistry, vol. 83, pp. 7198-7206, 2011/09/15 2011.

[5] P. R. Lewis, et al., "Recent advancements in the gasphase MicroChemLab," Sensors Journal, IEEE, vol. 6, pp. 784-795, 2006.

[6] R. Manginell, et al., "A Monolithically-Integrated $\mu \mathrm{GC}$ Chemical Sensor System," Sensors, vol. 11, pp. 6517 6532, 2011.

[7] B. C. Kaanta, et al., "A monolithically fabricated gas chromatography separation column with an integrated high sensitivity thermal conductivity detector," Journal of Micromechanics and Microengineering, vol. 20, p. 055016, 2010.

[8] S. Sreedharan Nair, et al., "A 2-Port Static Coated Micro Gas Chromatography Column with an Embedded Thermal Conductivity Detector," Sensors Journal, IEEE, pp. 1-1, 2011.

[9] B. Alfeeli and M. Agah, "Toward Handheld Diagnostics 
of Cancer Biomarkers in Breath: Micro Preconcentration of Trace Levels of Volatiles in Human Breath," Sensors Journal, IEEE, pp. 1-1, 2011.

[10] B. Bae, et al., "A fully-integrated MEMS preconcentrator for rapid gas sampling," 2007, pp. 14971500.

[11] S. Narayanan, et al., "A micro gas chromatography chip with an embedded non-cascaded thermal conductivity detector," Procedia Engineering, vol. 5, pp. 29-32, 2010.

[12] C. Lyons, et al., "A high-speed mass flow sensor with heated silicon carbide bridges," 1998, pp. 356-360.

[13] D. Cruz, et al., "Microfabricated thermal conductivity detector for the micro-ChemLab ${ }^{\mathrm{TM}}, "$ Sensors and Actuators B: Chemical, vol. 121, pp. 414-422, 2007.

[14] S. Frederico, et al., "Silicon sacrificial layer dry etching (SSLDE) for free-standing RF MEMS architectures," 2003, pp. 570-573.

[15] S. Reidy, et al., "High-performance, static-coated silicon microfabricated columns for gas chromatography," Analytical Chemistry, vol. 78, pp. 2623-2630, 2006.

\section{CONTACT}

*Dr. Masoud Agah, tel: +1-540-231-2653; agah@vt.edu 